\title{
PERFIL CONATIVO DE UNA MUESTRA DE NIÑOS DE LA CALLE DE CEUTA
}

CONATIVE PROFILE OF A SAMPLE OF STREET CHILDREN OF CEUTA

\author{
Sergio Cepero Espinosa* y Francisco Herrera Clavero** \\ Universidad de Granada
}

\section{RESUMEN ${ }^{1}$}

Esta investigación pretende conseguir dos objetivos: Primero, identificar el perfil conativo de una muestra de niños de la calle de la Ciudad Autónoma de Ceuta y, segundo, resaltar la necesidad de incorporar al estudio tradicional de las características específicas de los niños y jóvenes de la calle, otros aspectos que, a nivel mundial, comparten entre sí como grupo poblacional.

La información obtenida permite afirmar la existencia de dos perfiles bien diferenciados que aglutinan a los menores de la muestra. El primer perfil, agrupa a los menores que se "adaptan mejor" al ámbito institucional; mientras que, el segundo, recoge a aquellos que sufren mayor inadaptación socio-relacional, siendo los que más se resisten a cualquier tipo de tratamiento.

Palabras clave: Niños de la calle, perfil conativo, Ciudad Autónoma de Ceuta.

\begin{abstract}
This research seeks to achieve two goals: First, to identify the conative profile of a sample of street children from the Autonomous City of Ceuta and, secondly, to emphasize the need to incorporate to the traditional study of the specific characteristics of street children and youth, other aspects that, at world-wide level, share each other as a population group.

The obtained data allows us to affirm the existence of two differentiated profiles that cluster the children of the sample. The first profile groups the children who "adapt better" to the institutional scope

\footnotetext{
* Dr. Sergio Cepero Espinosa, con línea de investigación y publicaciones en el ámbito de los niños de la calle. Becario de Doctorado del Instituto de Estudios Ceutíes (2003-2005). Becario post-doctoral (2006-2008) del M.E.C. en los Estados Unidos (Columbia University, New York) en la investigación: "U.N. Millennium Project: The Millennium Villages Project". E-mail: filipenses2001@yahoo.es

** Dr. Francisco Herrera Clavero. Maestro, doctor en Filosofía y Ciencias de la Educación por la UNED, doctor en Psicología por la Universidad de Granada y catedrático de E.U. en Psicología Evolutiva y de la Educación de la Universidad de Granada, con líneas de investigación y numerosas publicaciones en el ámbito del aprendizaje, el currículo, la inmigración, la interculturalidad y la convivencia, y miembro numerario y de la Junta Rectora del Instituto de Estudios Ceutíes.
} 
and, the second one gathers those who undergo greater partner-relational maladjustment, these being the ones who better resist any type of treatment.

Key words: Street children, conative (socio-relational) profile, Autonomous City of Ceuta.

\section{Introducción}

El fenómeno de los niños de la calle no es algo nuevo en absoluto. Los niños han vivido y trabajado en las calles durante generaciones. Sin embargo, la escala actual del problema es mucho mayor de lo que pudiera haber sido nunca antes (Mcdonald y Garrow, 2000).

Según UNICEF existen más de 300 millones de niños que viven en las calles alrededor del mundo. Solamente en el mundo en desarrollo se estima que unos 100 millones de niños viven y trabajan en las calles: 40 millones en América Latina (Casa Alianza, 2000).

En la literatura internacional, la mayor parte de los investigadores sobre los niños y jóvenes de la calle, optan por dividir su estudio en dos vertientes: los niños de la calle en los países en desarrollo y desarrollados. Sin embargo, recientemente se está imponiendo una visión más internacional sobre el fenómeno en la que se examinan no sólo las características específicas o diferenciales que poseen estos colectivos, sino también los rasgos comunes que comparten.

El objetivo de este trabajo es obtener el perfil conativo (socio-comportamental) de una muestra de niños de la calle de Ceuta, mostrando características generales que puedan compartir con otros grupos de niños y jóvenes sin hogar (relaciones y estructura familiar, condiciones de vida, razones para abandonar el hogar, educación, sobreexplotación laboral infantil, etc.). No obstante, sólo las intervenciones que procesen información a nivel particular y global sobre este colectivo, lograrán resultados de éxito perdurables en el tiempo.

\section{Metodología}

\section{Muestra}

Está formada por cincuenta y tres niños procedentes de dos Centros de Menores y de un Piso Tutelado de Menores, todos ellos de Ceuta. Los niños tenían entre 9 y 17 años, siendo la media de edad de la muestra de 14,8 años. Sólo cinco niñas participaron en cada una de las pruebas, por lo que no es posible extrapolar ningún resultado referido a las diferencias de género; aunque para confirmarlo, se aplicó el análisis binomial para pruebas no paramétricas.

\section{Variables}

Factor I (ABUSO DOMÉSTICO INFANTIL/DUREZA DE LA VIDA EN LAS CALLES): Hace referencia a aquellos niños que abandonaron su hogar debido al abuso físico, psicológico y/o sexual sufrido en la intimidad de sus propios hogares, por sus padres o familiares cercanos. Una vez en las calles, tienen una muy alta involucración en conductas de riesgo tales como el abuso de drogas (cocaína y heroína), embarazo adolescente (inclu- 
yendo intentos de aborto), tráfico y venta de drogas; así como disfrute de la violencia en continuas peleas.

Factor II (HÁBITOS DE SUPERVIVENCIA EN LAS CALLES): Se refiere a aquellos menores que se sumergieron por completo en la cultura o el mundo de las calles. Por lo tanto, son niños y niñas acostumbrados a verse envueltos en todo tipo de peleas, robos y detenciones policiales. Asimismo, mantuvieron relaciones sexuales a edades muy tempranas; abusando de inhalantes, cannabis e incluso drogas de inyección.

Factor III (NIVEL ECONÓMICO Y RELACIONES FAMILIARES COMO PRECIPITADORES DE LA VIDA EN LAS CALLES): La pobreza y la desintegración del modelo familiar, llevan a estos niños y niñas a acudir a las calles en búsqueda de un futuro mejor. Es posible que para sobrevivir en las calles hayan recurrido a la mendicidad y que todavía mantengan algún contacto con su familia o sepan cuál es la situación de sus familiares.

Factor IV (SOBREEXPLOTACIÓN LABORAL INFANTIL): Referido a aquellos menores que fueron obligados por sus familias a trabajar, normalmente dentro de la economía informal, (venta ambulante, porteadores de mercancía en la frontera entre Marruecos y Ceuta, etc.). Apenas disfrutan de tiempo libre para descansar o estar con sus amigos, y tienen miedo a posibles represalias de sus jefes o de sus propias familias si dejaban su trabajo.

Factor V (ABANDONO DEL COLEGIO, TRABAJO INFANTIL Y RELACIONES SEXUALES PREMATURAS): Referido a los niños y niñas que por razones económicas tuvieron que trabajar en vez de asistir al colegio. Normalmente trabajaron por un tiempo dentro del sector tradicional (pastor, agricultor) a cambio de un poco de dinero y alojamiento. Después, vivieron en las calles, siendo víctimas en algunos casos de violaciones y manteniendo un elevado número de subsecuentes relaciones sexuales sin protección.

Factor VI (COMPROMISO Y PRÁCTICA DE LOS VALORES DE LA FE-CULTURA-RELIGIÓN): Son niños que conocen los "mandamientos" o "pilares" más importantes de su fe; dedican tiempo diario a la oración y asisten regularmente a la iglesia o mezquita.

Factor VII (VIDA EN LAS CALLES MOTIVADA POR RAZONES ECONÓMICAS, MALTRATO, ABANDONO U ORFANDAD: Estos menores fueron maltratados por sus familias; decidieron dejar sus casas para buscar un mejor futuro o, simplemente fueron desplazados al quedarse huérfanos. Han vivido sus vidas en desconfianza y completa soledad por lo que no guardan ningún recuerdo grato del pasado.

Factor VIII (INFLUENCIA DEL GRUPO DE IGUALES EN LA DECISIÓN DE VIVIR EN LAS CALLES / ADQUISICIÓN DE NUEVOS HÁBITOS Y COSTUMBRES): Se refiere a aquellos niños que se sintieron atraídos a la vida en las calles por la supuesta libertad de la que disfrutaban sus amigos que vivieron en las calles antes que ellos. Actualmente, están adquiriendo nuevos hábitos de disciplina y de estudio (aprendizaje del español) y declaran que mantienen buenas relaciones con amigos de otras culturas y religiones.

Factor IX (APRECIO POR LOS VALORES, LENGUA Y CULTURA PROPIAS): Son niños que conservan buenos recuerdos de su familia y país, viéndose obligados a abandonarlos por razones económicas. Se sienten orgullosos de su lengua materna y de las costumbres culturales que heredaron.

Factor X (SEXO DE SUPERVIVENCIA Y TRABAJO INFANTIL): Referido a los niños y niñas que conservan buenos recuerdos de sus familias y de su país; respetando su 
cultura-fe-religión. Lo más normal es que durante su tiempo en las calles trabajaran dentro del sector formal (subcontratación en talleres, construcción, hostelería) y que se han visto obligados a intercambiar sexo a cambio de dinero, comida o refugio.

\section{Instrumentos}

Dado que no existe ningún cuestionario estandarizado para este colectivo de niños, se construyó uno propio, basado en una profunda revisión teórica sobre el fenómeno de los niños de la calle y en la experiencia práctica diaria de convivencia que los autores mantuvieron con estos niños. El cuestionario está formado por datos de tipo sociológico y conativo en forma de escalas Likert (pentatipos) que consta de 132 ítems.

\section{Procedimiento}

Los niños y jóvenes que viven en las calles de cualquier país en desarrollo o desarrollado del mundo, se caracterizan por su desconfianza y difícil acceso. Por tal motivo, durante nueve meses, se contactó y convivió diariamente con los menores de la calle de la Ciudad Autónoma de Ceuta, a través de la Asociación Elín, entidad colaboradora en nuestra investigación.

Pasado este tiempo y gracias también a la colaboración de la Consejería de Bienestar Social de la Ciudad Autónoma de Ceuta, se pudo entrevistar a estos menores en varios centros de acogida. El proceso de recogida de datos comenzó en enero del año 2004 y concluyó a finales del mes de mayo de dicho año. En todo momento, los menores conocieron la naturaleza de los instrumentos y las condiciones de la investigación antes de la recogida de los datos y se tuvieron en cuenta también las condiciones deontológicas exigidas en la investigación humana.

\section{Análisis estadístico}

En primer lugar, se procedió a calcular la fiabilidad y validez factorial del cuestionario conativo. Del análisis factorial, surgieron los diez factores citados anteriormente. A continuación, se realizó un análisis de conglomerados $K$ medias, del que se obtuvieron los dos perfiles de niños de la calle de nuestra muestra.

\section{Resultados}

En las tablas 1, 2 y 3 aparecen los resultados referidos a la fiabilidad y validez del instrumento, así como el peso de cada conglomerado.

A través de los datos puestos de relieve por el programa estadístico SPSS-12, se confirma que, efectivamente, aparecen dos grupos de niños bien diferenciados (Gráfico 1). Un primer grupo, con un peso muestral de $36,30 \%$ que demuestra una buena adaptación a la vida en los centros de menores y que progresa hacia la realización de conductas pro-sociales. Son niños que no han sufrido abuso doméstico infantil ni, en gran medida, la dureza de 
TABLA 1: Fiabilidad del instrumento y análisis factorial de los datos (factores 1-5).

\begin{tabular}{|c|c|c|c|c|c|c|c|c|c|}
\hline \multicolumn{10}{|c|}{ Fiabilidad del Cuestionario Conativo (I) } \\
\hline Ítems & $\begin{array}{c}\text { Factor } \\
1\end{array}$ & Ítems & $\begin{array}{c}\text { Factor } \\
2\end{array}$ & Ítems & $\begin{array}{c}\text { Factor } \\
3\end{array}$ & Ítems & $\begin{array}{c}\text { Factor } \\
4\end{array}$ & Ítems & $\begin{array}{c}\text { Factor } \\
5\end{array}$ \\
\hline P116 & 0,890 & P118 & 0,808 & $\mathrm{P} 13$ & 0,613 & P90 & 0,770 & P95 & 0,698 \\
\hline P117 & 0,854 & P121 & 0,735 & P72 & 0,607 & P91 & $-0,764$ & P50 & $-0,594$ \\
\hline P114 & 0,814 & P124 & 0,731 & P10 & 0,563 & P93 & 0,671 & P87 & 0,580 \\
\hline P115 & 0,814 & P125 & 0,729 & P1 & 0,519 & P96 & $-0,600$ & P79 & 0,535 \\
\hline P23 & $-0,754$ & P105 & $-0,591$ & P3 & 0,503 & P97 & $-0,596$ & P126 & $-0,529$ \\
\hline P6 & 0,588 & P92 & $-0,539$ & P57 & 0,481 & P83 & $-0,433$ & P88 & 0,516 \\
\hline P18 & $-0,514$ & P123 & 0,516 & P84 & $-0,480$ & P68 & $-0,410$ & P86 & $-0,486$ \\
\hline P20 & 0,509 & P108 & 0,497 & P67 & $-0,474$ & $\mathrm{P} 120$ & $-0,402$ & P80 & 0,475 \\
\hline P62 & 0,484 & P122 & 0,477 & P74 & 0,471 & P94 & $-0,371$ & P81 & $-0,413$ \\
\hline P41 & $-0,433$ & P101 & 0,441 & P30 & 0,468 & P89 & 0,371 & P7 & $-0,406$ \\
\hline P132 & 0,423 & P78 & 0,430 & P65 & 0,453 & P100 & 0,275 & P55 & $-0,386$ \\
\hline P103 & 0,416 & P66 & 0,422 & P106 & 0,440 & P75 & 0,243 & P54 & $-0,363$ \\
\hline P128 & 0,404 & P77 & $-0,341$ & P102 & 0,408 & & & P111 & 0,323 \\
\hline P45 & $-0,367$ & P99 & 0,340 & P107 & 0,398 & & & P109 & $-0,304$ \\
\hline P69 & 0,358 & P71 & 0,320 & P14 & $-0,316$ & & & P113 & 0,296 \\
\hline P42 & 0,346 & & & & & & & P53 & 0,284 \\
\hline P119 & $-0,313$ & & & & & ficiont & Fiahi & & \\
\hline P73 & $-0,255$ & & & & & & & & \\
\hline
\end{tabular}

* Método de extracción: Análisis de componentes principales.

** Método de rotación: Normalización Varimax con Kaiser.

la vida en las calles (factor 1), no se han involucrado en numerosas conductas de riesgo para sobrevivir en las calles (factor 2), fueron las razones económicas, maltrato, abandono $\mathrm{u}$ orfandad las que principalmente los empujaron a vivir en las calles (factores 3 y 7), generalmente no han sido víctimas de la sobreexplotación laboral infantil (factores 4 y 10), ni tuvieron que abandonar el colegio para trabajar (factor 5). Asimismo, están comprometidos con su cultura-fe-religión (factor 6), no se dejaron influir por sus iguales para acudir a las calles (factor 8), aprecian los valores y la lengua propia de su cultura (factor 9) y, generalmente, no tuvieron que recurrir a la práctica de sexo de supervivencia (factor 10). Por el contrario, los menores del segundo perfil (peso muestral de 79,20\%) se caracterizan por su comportamiento opuesto en cada uno de los factores señalados; lo cual, los hace ser niños 
de difícil reinserción institucional y social, para los que es necesario emplear otro tipo de aproximaciones psico-pedagógicas.

TABLA 2: Fiabilidad del instrumento y análisis factorial de los datos (factores 6-10).

\begin{tabular}{|c|c|c|c|c|c|c|c|c|c|}
\hline \multicolumn{10}{|c|}{ Fiabilidad del Cuestionario Conativo (II) } \\
\hline Ítems & $\begin{array}{c}\text { Factor } \\
6\end{array}$ & Ítems & $\begin{array}{c}\text { Factor } \\
7\end{array}$ & Ítems & $\begin{array}{c}\text { Factor } \\
8\end{array}$ & Ítems & $\begin{array}{c}\text { Factor } \\
9\end{array}$ & Ítems & $\begin{array}{c}\text { Factor } \\
10\end{array}$ \\
\hline P25 & 0,830 & P8 & $-0,643$ & P27 & 0,713 & P51 & 0,679 & P39 & 0,730 \\
\hline P26 & 0,797 & P40 & $-0,601$ & P63 & $-0,578$ & P37 & 0,614 & P38 & 0,695 \\
\hline P24 & 0,766 & P5 & 0,550 & P35 & 0,549 & P28 & $-0,566$ & P44 & 0,567 \\
\hline $\mathrm{P} 22$ & 0,530 & P60 & 0,530 & P59 & 0,548 & P12 & $-0,543$ & P16 & $-0,456$ \\
\hline & & P4 & 0,473 & P32 & 0,547 & P34 & 0,482 & P110 & $-0,447$ \\
\hline & & P61 & $-0,473$ & $\mathrm{P} 127$ & 0,537 & $\mathrm{P} 130$ & $-0,458$ & $\mathrm{P} 112$ & 0,439 \\
\hline & & P70 & $-0,459$ & P49 & $-0,500$ & P56 & $-0,446$ & P82 & $-0,429$ \\
\hline & & P47 & 0,426 & P64 & $-0,490$ & P48 & 0,412 & P129 & 0,424 \\
\hline & & P11 & 0,400 & P46 & 0,410 & P31 & 0,399 & & \\
\hline & & P104 & $-0,330$ & P58 & $-0,387$ & P52 & 0,397 & & \\
\hline & & P85 & 0,328 & P98 & $-0,386$ & P9 & $-0,343$ & & \\
\hline & & $\mathrm{P} 2$ & 0,276 & P19 & 0,314 & P21 & $-0,331$ & & \\
\hline & & P131 & 0,218 & P15 & $-0,296$ & P76 & $-0,262$ & & \\
\hline & & & & & $\begin{array}{r}\mathrm{Cc} \\
\text { "Alp }\end{array}$ & $\begin{array}{l}\text { ficiente } \\
\text { a" de C }\end{array}$ & $\begin{array}{l}\text { e Fiabili } \\
\text { mbach }=\end{array}$ & $\begin{array}{l}\text { d: } \\
, 538\end{array}$ & \\
\hline
\end{tabular}

* Método de extracción: Análisis de componentes principales.

** Método de rotación: Normalización Varimax con Kaiser. 
TABLA 3: Puntuaciones y peso muestral de los conglomerados finales.

\begin{tabular}{|c|c|c|}
\hline \multirow{2}{*}{$\begin{array}{l}\text { Puntuaciones y peso muestral de los } \\
\text { Factores conativos }\end{array}$} & \multicolumn{2}{|c|}{$\begin{array}{l}\text { Conglomerados finales para } \\
\text { los dos perfiles }\end{array}$} \\
\hline & Perfil 1 & Perfil 2 \\
\hline $\begin{array}{l}\text { Puntuación Factor 1: Abuso doméstico infantil y dureza de la } \\
\text { supervivencia en las calles. }\end{array}$ & $-1,79917$ & 0,25346 \\
\hline Puntuación Factor 2: Hábitos de supervivencia en las calles. & $-0,53235$ & 0,13030 \\
\hline $\begin{array}{l}\text { Puntuación Factor 3: Nivel económico y relaciones familiares } \\
\text { como precipitadores de la vida en las calles. }\end{array}$ & 0,33061 & $-0,05701$ \\
\hline Puntuación Factor 4: Sobreexplotación laboral infantil & $-0,29962$ & 0,03863 \\
\hline $\begin{array}{l}\text { Puntuación Factor } 5 \text { : Abandono del colegio, trabajo infantil y } \\
\text { relaciones sexuales prematuras. }\end{array}$ & $-0,39743$ & 0,05184 \\
\hline $\begin{array}{l}\text { Puntuación Factor 6: Compromiso y práctica de los valores de la } \\
\text { fe-cultura-religión. }\end{array}$ & 0,24101 & $-0,02232$ \\
\hline $\begin{array}{l}\text { Puntuación Factor 7: Vida en las calles motivada por razones } \\
\text { económicas, maltrato, abandono u orfandad (sentimientos de } \\
\text { soledad). }\end{array}$ & 0,33015 & $-0,03729$ \\
\hline $\begin{array}{l}\text { Puntuación Factor 8: Influencia del grupo de iguales en la } \\
\text { decisión de vivir en las calles/adquisición de nuevos hábitos y } \\
\text { costumbres }\end{array}$ & $-0,22122$ & 0,08387 \\
\hline $\begin{array}{l}\text { Puntuación Factor 9: Aprecio por los valores, lengua y cultura } \\
\text { propias. }\end{array}$ & 0,07654 & 0,01775 \\
\hline $\begin{array}{l}\text { Puntuación Factor 10: Sexo de supervivencia trabajo infantil y } \\
\text { deseos de futuro. }\end{array}$ & $-0,45460$ & 0,06387 \\
\hline Peso muestral: & $36,30 \%$ & $63,70 \%$ \\
\hline
\end{tabular}

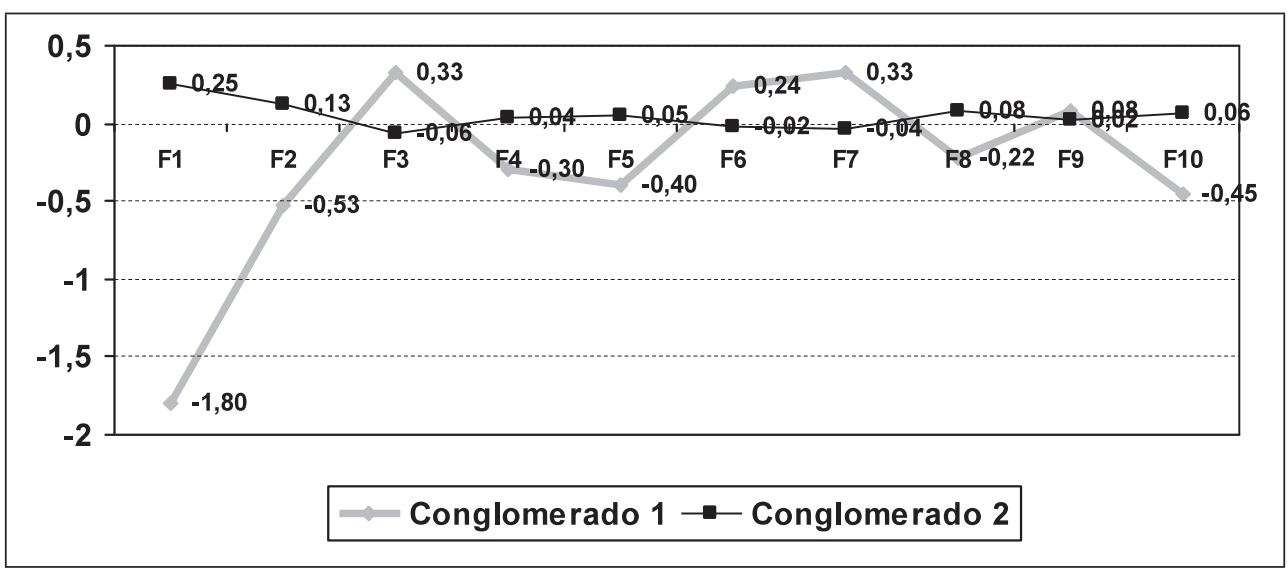

GRÁFICO 1.

Distribución de cada uno de los perfiles de la muestra. 


\section{Discusión}

1. Las condiciones sociales y situacionales tienen gran importancia en la formación de actitudes e influyen como factores de riesgo o factores protectores en la conducta de la persona (Ibáñez, Senra y Del Río, 1998). Los niños y jóvenes que viven en las calles, comparten dos características básicas: carecen de lugar de residencia y no son reconocidos por su comunidad. Por tanto, no suelen tener una red social que les sirva de apoyo (familia, amigos, etc.); cuentan con limitados recursos económicos y, muchas veces, para sobrevivir realizan actividades informales e ilegales (Solarz y Bogat, 1990, citados en Van Der Ploeg y Scholte, 1997).

Este proceso de aislamiento social comienza en la familia, reforzándose cuando el joven abandona la escuela y se ve rechazado por su grupo de iguales. Por ejemplo, Van der Ploeg et al. (1991 citados en Van Der Ploeg y Scholte, 1997), encontraron que el 73\% de los jóvenes de su muestra holandesa nunca había contactado con sus padres y el 21\% sólo mantenía contactos ocasionales; el 51\% nunca contactó con sus madres y el $31 \%$ sólo lo hizo ocasionalmente. El 17\% reconoció no tener ningún amigo y el 80\% afirmó no tener ningún amigo íntimo.

En el caso de los menores de nuestra muestra de Ceuta, encontramos porcentajes similares: el $52,1 \%$ de los niños de la muestra no ha vuelto a mantener el contacto con sus familias desde que abandonaron su hogar y el 39,6\% sólo ha mantenido contactos familiares ocasionales. De igual forma, un $46,5 \%$ declaró no tener ninguna persona a la que considerar amigo.

2. Investigaciones empíricas sobre los jóvenes sin hogar demuestran que la vida en las calles correlaciona significativamente con las actividades delictivas. El 71\% de los jóvenes sin hogar canadienses que participaron en la investigación de Kufeldt y Nimmo (1991, citados en Van Der Ploeg y Scholte, 1997), reconocieron estar de alguna forma asociados con actividades delictivas; medio principal de supervivencia para más de la mitad de la muestra. Conforme aumentaba su tiempo de vida en las calles, los jóvenes realizaban mayor número de actividades ilegales.

De forma paralela, en nuestra muestra son los menores que llevan más tiempo viviendo en la ciudad (cinco o más años, el $27 \%$ ) y que por lo tanto tienen quince, dieciséis o diecisiete años, los que verdaderamente tienen una opinión negativa sobre el trato policial recibido. También, son los que se han visto involucrados en mayor número de peleas y detenciones policiales $(17 \%)$, manteniendo niveles de consumo de drogas "legales" y "blandas" altos o muy altos $(17 \%$ y $24,5 \%)$ y habiendo estado mayor número de noches en comisarías $(43,4 \%)$.

3. Las investigaciones muestran que los jóvenes sin hogar abusan del alcohol y de las drogas a un nivel 3 o 4 veces mayor que sus iguales que asisten a la escuela; mientras que, para algunas drogas, como cocaína, heroína y LSD, los niveles de abuso son al menos 10 veces superiores a los de su grupo de iguales escolarizados (Feitel et al., 1992; Fors y Rojek, 1990; McKirman y Johnson, 1986; Robertson et al., 1989 citados en Van Der Ploeg y Scholte, 1997).

La iniciación temprana en el consumo de drogas se asocia con una escalada mayor de drogadicción, ya sea con las mismas drogas o con otras diferentes (National Institute On Drug Abuse, 2003). En nuestra muestra, también parece existir una "evolución" 
en el consumo de diferentes drogas que aumenta según la edad. Los niños de menor edad comienzan con el pegamento industrial entre los seis y los ocho años $(18,2 \%)$, siendo el período de edad de mayor consumo entre los nueve y once años $(45,5 \%)$. Por otro lado, el mayor porcentaje de niños que consumen drogas "legales" se concentra en la franja de edad de los nueve a once años $(58,1 \%)$; siendo el grupo de edad más representativo para el consumo de drogas "blandas" y "pastillas" el de doce y catorce años (47,8\% y 61,5\%, respectivamente). Los casos registrados sobre consumo de drogas "duras" se producen entre los doce y catorce años. El único menor que reconoció haber usado "drogas de inyección", se inyectó "heroína" entre los quince y los diecisiete años.

4. Finalmente, considerando los dos perfiles de niños de nuestra muestra, no podemos aplicar una única intervención psicoeducativa con ellos, ya que siempre quedarán menores a los que no alcancemos y a los que daremos por "imposibles". Es necesario diseñar programas de intervención "a medida" con cada uno de los dos perfiles de menores. Para ello, deben proponerse programas integrales e interdisciplinares, también conocidos como "multi-factoriales", al considerar simultáneamente todos los factores de riesgo que afectan a las áreas de socialización del joven y ofrecerle una "mezcla equilibrada" de servicios sociales efectivos y comprometidos con su persona (Van Der Ploeg y Scholte, 1997). Los programas de mayor éxito incluyen intervenciones a largo plazo, con revisiones periódicas en las que se evalúan si los servicios ofrecidos realmente contribuyen a mejorar la vida de los jóvenes (Van Der Ploeg y Scholte, 1997). Es necesario que se involucren en esfuerzos de prevención a todas las instituciones, organizaciones (públicas y privadas) y personas que regulan la vida social y económica de la comunidad (Van Der Ploeg y Scholte, 1997). Algunas de estas actuaciones deberían encaminarse preferentemente, en primer lugar, a la adquisición y dominio de la lengua española (adquisición, más que aprendizaje, a través de métodos funcionales aplicados a las necesidades e intereses), a la mejora de interrelaciones sociales y a la resolución de conflictos de estos niños, tales como (Herrera y Ramírez, 2005):

- Técnicas de grupo y de dinámica de grupos: (Burns, 1979; Hostie, 1994; Pallarés, 1990).

- Entrenamiento en habilidades sociales (González y Pelechano, 1996; Monjas, 1993 y 1999; Trianes y Muñoz, 1994).

En este mismo contexto, algunas de las propuestas más destacables que tratan de conectar al niño con su realidad social (conociéndola, comprendiéndola y valorándola), siguiendo un enfoque próximo-distal, son (Herrera y Ramírez, 2005):

- Programa de Enseñanza de Habilidades Sociales (Michelson, Sugai, Wood y Kazdin, 1987).

- Programa de Aprendizaje Estructurado (Goldstein, Sáenz y Martínez, 1989).

- Ratones, Dragones y Seres Humanos Auténticos (García y Magaz, 1992a).

- Aprendiendo a Comunicarse con Eficacia (García y Magaz, 1992b).

- Programa de Refuerzo de las Habilidades Sociales (Vallés, 1994).

- Programa de Enseñanza de Habilidades Sociales en Educación Primaria (Álvarez, 1999). 
- Programa Integrado de Entrenamiento en Habilidades Interpersonales para el Ciclo Medio (González y Pelechano, 1996).

- Programa de Entrenamiento en Fases de Solución de Problemas para el Ciclo Superior/ESO (Joly y Pelechano, 1996).

- Programa de Enseñanza de Habilidades de Interacción Social (Monjas, 1999).

\section{Referencias bibliográficas}

Alonso, E. (1999). Intervención para la mejora del autoconcepto en alumnos de educación secundaria. Tesis Doctoral. Las Palmas de Gran Canaria: Universidad de Las Palmas de Gran Canaria. Servicio de Publicaciones y Producción Documental.

Alonso, E. (2001). Intervención para la mejora del autoconcepto en alumnos de educación secundaria. Las Palmas de Gran Canaria: Universidad de Las Palmas de Gran Canaria. Servicio de Publicaciones y Producción Documental.

Álvarez, J. (1999). Programa de Enseñanza de Habilidades Sociales en Educación Primaria. Málaga: Aljibe.

Burns, R. B. (1979). The self concept. Theory, measurement, development and behaviour. New York: Logman.

Casa Alianza. (2000). Viviendo en las calles. Extraído el 12 de noviembre de 2004, de http://www. casa-alianza.org/

García, E. M. y Magaz, A. (1992a). Ratones, dragones y seres humanos auténticos. Madrid: CEPE

García, E. M. y Magaz, A. (1992b). Aprendiendo a comunicarme con eficacia. Entrenamiento en habilidades de comunicación asertiva. Madrid: CEPE

Goldstein, A. P., Sáenz, J. y Martínez, A. (1989). Habilidades sociales y autocontrol en la adolescencia. Barcelona: Martínez Roca.

González, P. y Pelechano, V. (1996). "Programa Integrado de Entrenamiento en Habilidades Interpersonales en el Ciclo Medio". En Pelechano, V. (Dir.). Habilidades Interpersonales. Teoría Mínima y Programas de Intervención. Vol. II. Valencia: Promolibro.

Herrera, F. y Ramírez, M. I. (2005). "Pluriculturalidad, multiculturalidad e interculturalidad". En Castellano, D., Gil, A. y Serrano, P.: Mujeres. El análisis (pp. 283-311). Colección año 2468. La mediación social. Universidad Jaime I: Fondo Social Europeo. Proyecto Equal.

Hostie, R. (1994). Técnicas de dinámicas de grupos. Barcelona: Herder.

Ibáñez, P., Senra, M. P. y Del Río, M. (1998). "Factores socioprofesionales del drogodependiente". Revista española de orientación y psicopedagogía, Vol. 9, N 16, 1998, pp. 243-256.

Joly, P. y Pelechano, V. (1996). Programa de Entrenamiento en Fases de Solución de Problemas para el Ciclo Superior/ESO. Valencia: Promolibro.

Machargo, J. (1989). El profesor y el autoconcepto de sus alumnos. Madrid: Escuela Española.

Mcdonald, P. y Garrow, E. (2000). Reaching children in need: What's being done-what you can do. Eastbourne, Inglaterra: Kingsway Publications.

Michelson, L., Sugai, D. P., Wood, R. P. y Kazdin, A. E. (1987). Las habilidades sociales en la infancia. Evaluación y tratamiento. Barcelona: Martínez Roca.

Monjas, M. I. (1993). Programa de enseñanza de habilidades de interacción social. Para niños y niñas en edad escolar. Valladolid: M.I.M.C. 
Monjas, M. I. (1999). Programa de enseñanza de habilidades de interacción social (PEHIS) para niños y niñas en edad escolar. Madrid: CEPE.

National Institute On Drug Abuse (2003). Preventing drug use among children and adolescents. A research-based guide for parents, educators, and community leaders. Extraído el 12 de noviembre de 2004, de http://www.drugabuse.gov

Pallarés, M. (1990). Técnicas de grupo para educadores. Madrid: ICCE.

Pelechano, V. y González Leandro, P. (1996a). "Programa de entrenamiento en fases de solución de problemas". En V. Pelechano (Dir.). Habilidades Interpersonales. Teoría Mínima y Programas de Intervención. Vol. II. Valencia: Promolibro.

Pelechano, V. y González, P. (1996b). "Programa de entrenamiento en dimensiones de solución de problemas interpersonales". En V. Pelechano (Dir.). Habilidades Interpersonales. Teoría Minima y Programas de Intervención. Vol. II. Valencia: Promolibro.

Trianes, M. V. y Muñoz, A. (1994). Programa de Educación Social y Afectiva. Málaga: Puerta Nueva, Delegación de Educación y Cultura.

Vallés, A. (1994). Programas de refuerzo de las habilidades sociales. Madrid: EOS.

Van Der Ploeg, J. y Scholte, E. (1997). Homeless youth. Working with children and adolescents series. London: SAGE Publications.

Fecha de recepción: 20-04-06

Fecha de revisión: 26-06-07

Fecha de aceptación: 07-11-07 